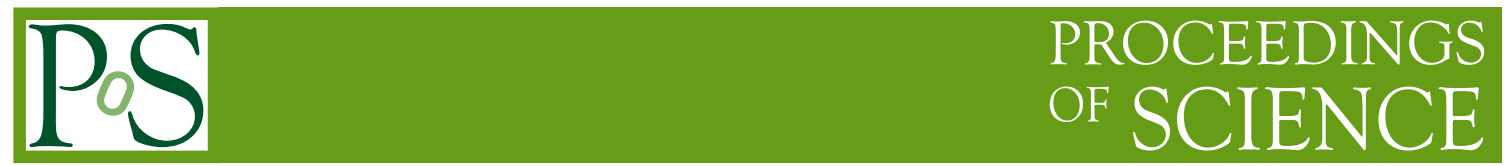

\title{
Inclusive Semileptonic D Decays and the Heavy Quark Expansion
}

\author{
J. F. Kamenik* \\ J. Stefan Institute, Jamova 39, P. O. Box 3000, 1001 Ljubljana, Slovenia \\ E-mail: jernej.kamenikeijs.si
}

\begin{abstract}
The recently measured inclusive semileptonic $D_{(s)}$ decays are sensitive probes of Weak Annihilation. Both the total widths and the lepton energy spectral moments can be studied theoretically using the heavy quark expansion. The consistency and behavior of the perturbative series has recently been analyzed in several quark mass schemes. The analysis of Cleo data shows no clear evidence of Weak Annihilation, and allows to put bounds on its relevance in charmless $B$ semileptonic decays.
\end{abstract}

Flavor Physics and CP Violation - FPCP 2010

May 25-29, 2010

Turin, Italy

\footnotetext{
* Speaker.
} 


\section{Introduction}

The value of $\left|V_{u b}\right|$ preferred by current global analyses of CKM data is about $15 \%$ smaller than the one extracted from inclusive charmless semileptonic $B$ decays [1]. Though not very significant, the discrepancy has prompted a reexamination of the sources of theoretical uncertainty in the inclusive determination [2]. Weak Annihilation (WA) contributions are generally considered an important source of uncertainty in the Operator Product Expansion (OPE) that describes the inclusive $B$ decays [3], and affect especially the high $q^{2}$ and lepton endpoint analyses. They appear in the OPE as $1 / m_{b}^{3}$ corrections involving the matrix elements of dimension- 6 four-quark operators, and affect both the total $B \rightarrow X_{u} \ell \bar{v}$ decay rate and the charged lepton energy spectrum $[4,5]$.

It was noted in refs. $[6,7,8]$ that the WA matrix elements that enter $B \rightarrow X_{u} \ell \bar{v}$ decay can be constrained via the semileptonic decays of $D$ and $D_{s}$ mesons, using heavy quark symmetry. Several authors have attempted to extract information on WA contributions from the measured total semileptonic rates of $D^{0, \pm}$ and $D_{s}$, most recently in $[9,10,11]$. For instance, one may attribute the observed differences in $D^{ \pm, 0}$ and $D_{s}$ semileptonic widths [12]

$$
\Gamma\left(D^{+} \rightarrow X e^{+} v\right) / \Gamma\left(D^{0} \rightarrow X e^{+} v\right)=0.985(28), \quad \Gamma\left(D_{s}^{+} \rightarrow X e^{+} v\right) / \Gamma\left(D^{0} \rightarrow X e^{+} v\right)=0.828(57)
$$

to the valence spectator quark WA contributions in $D_{s}$ decays, since they are Cabibbo suppressed in the $D^{ \pm}$case and completely absent in $D^{0}$ decays [10]. However, additional contributions to this difference arise from $S U(3)$ breaking in the matrix elements of all dimension 5 and 6 operators, that contribute significantly to the total rates [6]. Furthermore, such analysis leaves the non-valence WA contributions unconstrained. For these to be extracted, a reliable computation of the total semileptonic widths is needed. Since the charm mass is not particularly large compared with nonperturbative QCD scales, such computation suffers from both large perturbative and $1 / m_{c}^{n}$ corrections, limiting the precision with which WA can be studied in this way [11].

A complementary strategy is to consider also the moments of the lepton energy spectra in the OPE and compare them with recent experimental results from Cleo [12]. Not only are the moments free of the strong dependence on the charm quark mass and its associated uncertainty, but their perturbative and non-perturbative corrections tend to cancel as well. Moreover, since WA is expected to dominate the spectrum endpoint, leptonic moments might be more sensitive to WA contributions than the total rate ${ }^{1}$. A study of moments in $D$ semileptonic decays is also instrumental for a critical reassessment of the OPE in charm decays many years after [15], in view of the recent experimental results and of the successful application to $B$ semileptonic decays.

\section{Experimental data}

Recently the Cleo Collaboration has measured the electron spectra of inclusive semileptonic $D^{ \pm, 0}$ and also $D_{s}$ decays with a lower cut on the electron momentum in the lab frame of $p_{e}>0.2$ $\mathrm{GeV}$ [12]. They extract the total decay rates by extrapolating the spectra over the remaining phasespace, using a theoretically modeled sum over known exclusive modes. Unfortunately, they do not provide higher leptonic energy moments.

\footnotetext{
${ }^{1}$ The role of WA in $D_{s}$ exclusive hadronic and semileptonic decays has recently been discussed in [13].
} 
In [14] the moments without cuts have been extracted by first extrapolating the measured spectra towards $p_{e}=0$ using OPE inspired extrapolation formulae and then boosting the results from the laboratory to the $D$ rest-frame. Within the stated uncertainties there is no sign of a difference between the moments of $D_{s}$ and $D^{0, \pm}$. This is at odds with what one would naively expect from what is observed in the rates [12]. However, as mentioned in the Introduction, the concurring contribution of $\mathrm{SU}(3)$ violation in the matrix elements of higher dimensional operators might provide a partial explanation. In order to reliably extract possible WA contributions from the measured total semileptonic rates of $D$ and $D_{s}$ mesons [12], one would therefore need to estimate the size of $S U(3)$ violation in all these matrix elements. In the case of normalized moments, on the other hand, some of the leading power corrections cancel out and one might be more directly sensitive to WA contributions.

\section{Inclusive semileptonic charm decays in the OPE}

The perturbative corrections to the total rate as well as moments of some kinematic distributions in semileptonic $b$ decays are now known through $O\left(\alpha_{s}^{2}\right)$ [16]. In the case of semileptonic charm decays, however, only the $O\left(\alpha_{s}\right)$ [17] and $O\left(\beta_{0} \alpha_{s}^{2}\right)$ corrections are readily available using [18]. For what concerns the power corrections, explicit expressions for the leptonic spectrum at $O\left(1 / m_{c}^{2}\right)$ can be found in the last paper of [3], while the $O\left(1 / m_{c}^{3}\right)$ contributions have recently been computed [14] from the form factors given in [19]. All present analyses neglect $O\left(1 / \mathrm{m}_{c}^{4}\right)$ corrections [20] and $O\left(\alpha_{s} / m_{c}^{2}\right)$ corrections, for which only the $\mu_{\pi}^{2}$ contribution to the semileptonic rate is known.

The total moments from the lepton energy spectra can be calculated in terms of $x \equiv 2 E_{e} / m_{c}$, where $E_{e}$ is the electron energy in the $D$ meson inertial frame. In [14] only the total leptonic moments, without lower cuts on the lepton energy have been considered. At present level of precision, the lepton mass can be safely neglected. Then, total semileptonic rate and higher unnormalized leptonic energy moments are defined as

$$
\begin{aligned}
\Gamma^{(n)} \equiv \int_{0}^{(1-r)} \frac{d \Gamma}{d x} x^{n} d x= & \frac{G_{F}^{2} m_{c}^{5}}{192 \pi^{3}}\left|V_{c s}\right|^{2}\left[f_{0}^{(n)}(r)+\frac{\alpha_{s}}{\pi} f_{1}^{(n)}(r)+\frac{\alpha_{s}^{2}}{\pi^{2}} f_{2}^{(n)}(r)+\frac{\mu_{\pi}^{2}}{m_{c}^{2}} f_{\pi}^{(n)}(r)+\frac{\mu_{G}^{2}}{m_{c}^{2}} f_{G}^{(n)}(r)\right. \\
& \left.+\frac{\rho_{L S}^{3}}{m_{c}^{3}} f_{L S}^{(n)}(r)+\frac{\rho_{D}^{3}}{m_{c}^{3}} f_{D}^{(n)}(r)+\frac{32 \pi^{2}}{m_{c}^{3}} B_{\mathrm{WA}}^{(n) s}\right],
\end{aligned}
$$

where $r=m_{s}^{2} / m_{c}^{2}, \alpha_{s} \equiv \alpha_{s}\left(m_{c}\right)$, and $\mu_{\pi, G}^{2}, \rho_{D, L S}^{3}$ are the $D$ meson matrix elements of the dimension 5 and 6 local operators appearing in the OPE. In addition, there are also Cabibbo suppressed contributions, which can be included in the analysis by using Eq. (3.1) in the limit $r \rightarrow 0$ and by replacing $V_{c s}$ with $V_{c d}$ and similarly $B_{\mathrm{WA}}^{(n), s}$ with $B_{\mathrm{WA}}^{(n), d}$. They contribute to the total rate at the level of $5 \%$, but their effect is highly suppressed in the normalized moments, with the possible exception of WA contributions.

The WA contributions to to the $n$-moment appear at the order $1 / m_{c}^{3}$ and the $B_{\mathrm{WA}}^{(n)}$ can be identified with the matrix elements of the dimension- 6 four quark operators

$$
\mathscr{O}_{1}^{Q q}=\bar{Q} \gamma_{\mu}\left(1-\gamma_{5}\right) q \bar{q} \gamma^{\mu}\left(1-\gamma_{5}\right) Q \quad \text { and } \quad \mathscr{O}_{2}^{Q q}=\bar{Q}\left(1-\gamma_{5}\right) q \bar{q}\left(1-\gamma_{5}\right) Q
$$


where $Q$ and $q$ are the heavy and light quark flavor indices respectively, entering charm meson decay rates

$$
\begin{aligned}
B_{\mathrm{WA}}^{c q}\left(\mu_{\mathrm{wA}}\right) & =\frac{1}{2 m_{D}}\left\langle D\left|\mathscr{O}_{2}^{c q}-\mathscr{O}_{1}^{c q}\right| D\right\rangle \\
& =\frac{1}{2} m_{D} f_{D}^{2}\left(B_{D, 2}^{q}-B_{D, 1}^{q}\right),
\end{aligned}
$$

where $B_{D, i}^{q}\left(\mu_{\mathrm{WA}}\right)$ parameterize the deviation from the factorization approximation at the renormalization scale $\mu_{\mathrm{WA}}: B_{\mathrm{WA}}^{Q q}$ vanishes in the limit of factorization. In physical observables, the implicit $\mu_{\mathrm{WA}}$ dependence cancels against the explicit scale dependence of the $f_{D}^{(n)}$ terms. A change of $\mu_{\mathrm{WA}}$ therefore shifts part of the Darwin operator contribution into the WA contribution, with important consequences for the error analysis. For values of $\mu_{\mathrm{WA}}$ below $1 \mathrm{GeV}$ the size of the $\rho_{D}^{3}$ coefficient in the width is comparable to that of other power corrections [14].

Neglecting small Cabibbo suppressed and isospin-violating effects, the WA contributions to the decays of charmed mesons involving different spectator quarks can be described in terms of just two distinct contributions, which can be identified with the valence and non-valence WA terms involving the $s$ quark. The first one, $B_{\mathrm{WA}}^{c s}\left(D_{s}\right)$, only contributes to the $D_{s}$ decays, while the second one, $B_{\mathrm{WA}}^{c s}(D)$, contributes equally to $D^{+}$and $D^{0}$ decays. In the flavor $S U(3)$ limit, the two contributions correspond to the sum of isotriplet $\left(\Delta B_{\mathrm{WA}}^{c}\right)$ and isosinglet $\left(B_{\mathrm{WA}}^{c}\right)$, and to the isosinglet contribution, respectively.

As it was recognized long ago [4], WA is localized at the endpoint of the lepton energy spectrum, and can be approximately expressed by a delta function at the partonic endpoint. In this case, one would expect $B_{\mathrm{WA}}^{(n)}=B_{\mathrm{WA}}^{c s}$, for all $n$, up to small $\mathscr{O}(r)$ effects. In fact, gluon bremsstrahlung and hadronization effects are expected to smear the WA contribution over a region of electron energy around the partonic threshold $\left(m_{c}^{2}-m_{s}^{2}\right) / 2 m_{c}$. The size and shape of the smearing may affect the various integrals $B_{\mathrm{WA}}^{(n)}$ differently and cannot be predicted, although one expects a small perturbative tail to emerge away from the endpoint. Clearly, smearing towards smaller $E_{e}$ tends to suppress the WA contributions to higher lepton energy moments, in which case one expects $B_{\mathrm{WA}}^{(n+1)} \lesssim B_{\mathrm{WA}}^{(n)}$. Indeed, the WA distributions spread over a region of electron energies of $\mathscr{O}\left(\Lambda_{Q C D}\right)$ [21]. We are primarily interested in a determination of the leading matrix element, $B_{\mathrm{WA}}^{(0)}$, i.e. the zeroth moment of the WA distribution, from $B_{\mathrm{WA}}^{(n)}$ : the non-negligible smearing of WA may lead to a model-dependent dilution. In order to quantify this effect on the parameters $B_{\mathrm{WA}}^{(n)}$, a number of smearing distributions have been considered [14]. In general the dilution is a moderate $\mathscr{O}(20 \%)$ effect. However, distributions characterized by a longer tail would lead to a stronger dilution, which may also be enhanced in the normalized moments.

\section{Results and Discussion}

Before attempting to extract WA from the measured semileptonic rates and spectra, one needs to check whether the OPE at $\mathscr{O}\left(1 / m_{c}^{3}\right)$ describes the experimental data in a satisfactory way. In particular, the perturbative corrections encoded in $f_{1,2}^{(n)}$ depend on the choice of scheme for $m_{c}$ (and the OPE parameters). The perturbation series for the total rate is ill-behaved when the rate is expressed in terms of the charm quark pole or $\overline{\mathrm{MS}}$ mass [11]. The bad behavior of these perturbation 
series arises from a poor choice for the heavy quark mass. A better behaved series is obtained by using a threshold mass scheme, like the kinetic [22], the PS [23], or the 1S [24] mass schemes, see [2] for a review. It was first noted in [25] that the perturbation series for the total rate in the $1 \mathrm{~S}$ scheme for $m_{c}$ is reasonably well-behaved. On the other hand, well behaved perturbative series in PS or kinetic mass schemes are only recovered for factorization scales considerably lower than the charm quark mass.

The dominant parametric uncertainty to the rate comes from extracting the charm quark mass from other physical quantities, such as moments of B decay spectra. Since the charm quark mass is an intermediate quantity which is not required for analysis of WA, it has been proposed in [11] to minimize this source of theoretical uncertainty by bypassing any choice of charm mass scheme, and instead directly relate the semileptonic $D$ decay widths to the values of $m_{b}$ and $\Delta=m_{b}-m_{c}$ extracted from a global fit to B decay spectra.

A determination of both valence and non-valence WA from the D semileptonic widths using $m_{b}$ and $\Delta$ in $1 \mathrm{~S}$ scheme [11] translates into $\Delta B_{\mathrm{WA}}^{c}=-0.0015(9) \mathrm{GeV}^{3}$ for the valence and $B_{\mathrm{WA}}^{c}=0.0036(5) \mathrm{GeV}^{3}$ for the isosinglet contribution. If one instead uses the kinetic scheme for the charm mass and the OPE parameters as extracted from the global fit to B decay spectra and translated to a lower kinetic scale $\mu_{\text {kin }}=0.5 \mathrm{GeV}$, one obtains from the rates at $\mu_{\mathrm{WA}}=0.8 \mathrm{GeV}$ : $\Delta B_{\mathrm{WA}}^{c}=-0.0014(12) \mathrm{GeV}^{3}$ and $B_{\mathrm{WA}}^{c}=-0.001(3) \mathrm{GeV}^{3}$ [14]. The two estimates for the valence contribution are perfectly compatible with each other. On the other hand, the non-valence contribution estimates differ significantly both in their central values as well as in their error estimates. The difference is presumably due to various sources: $i)$ the charm mass and OPE parameters in the two analyses, although both taken from the global B decay fits, are translated to the charm sector differently, which may lead to sizable differences in charm but not in bottom decays; ii) in [14], additional uncertainty due to possible $S U(3)$ violation is attributed to the OPE parameters thus inflating the final error estimates on WA iii) the method of [11] implies the use of $\alpha_{s}\left(m_{b}\right)$, and might underestimate the perturbative corrections. The discrepancy between the two determinations provides indeed additional motivation for using the moments to constrain WA as they are less sensitive to power and perturbative corrections.

The lepton moments indeed receive smaller perturbative corrections than the total rate, while they are more sensitive to WA and the Darwin term [14]. As for the $m_{c}$ dependence, since $\left\langle E^{n}\right\rangle$ scales like $m_{c}^{n}$, the lowest moments are less sensitive to the value of the charm mass than the width, which is proportional to $m_{c}^{5}$. Working in the kinetic scheme, the dominant source of parametric uncertainty in the first and the second leptonic energy moment is by far $\rho_{D}^{3}$. Varying all the input parameters within conservative ranges and comparing the theoretical predictions for both the rates and the first two moments with experimental values, the values of $B_{\mathrm{WA}}^{(0,1,2) s}$ have been extracted in [14]. At the estimated theoretical precision, all the extracted WA contributions are consistent with zero. The obvious implication is that the OPE describes all the data reasonably well. Compatible results have been obtained with kinetic scales between $\mu_{\text {kin }}=0.5-0.8 \mathrm{GeV}$ and also using the $1 \mathrm{~S}$ scheme for the charm mass. Although the apparent convergence of the perturbative expansion for the rate and variance changes, the estimates and errors of the WA contributions are very similar.

As already mentioned in the previous Section, WA are expected to be concentrated at the end-point, in which case one can combine the $D^{0,+}$ results to obtain the isosinglet $B_{\mathrm{WA}}^{(0)}\left(\mu_{\mathrm{WA}}=\right.$ $0.8 \mathrm{GeV})=-0.0003(15) \mathrm{GeV}^{3}$. In the worst case, the reduced sensitivity due to the WA smearing 

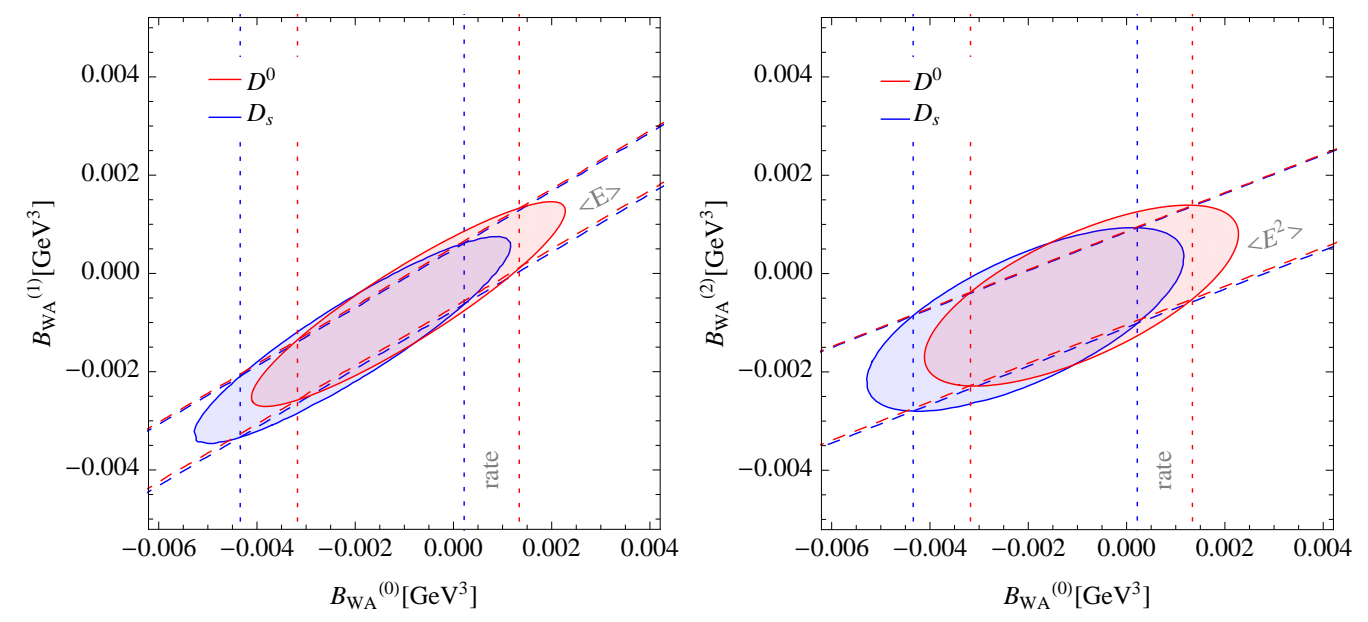

Figure 1: Combined constraints on $B_{\mathrm{WA}}^{(i), s}$ from the total semileptonic rates of $D^{0}$ and $D_{s}$ and the first two leptonic energy moments from [14].

functions is about $60 \%$, which leads to

$$
B_{\mathrm{WA}}^{c}\left(\mu_{\mathrm{WA}}=0.8 \mathrm{GeV}\right)=-0.0003(25) \mathrm{GeV}^{3},
$$

where, in order to be conservative, the dilution error has been combined linearly, without changing the central value.

One can also look for indications of WA dilution by extracting $B_{\mathrm{WA}}^{(0,1,2)}$ separately, from a combined fit to the moments and the total rates. The results for $D^{0}$ and $D_{s}$ decays are shown in figure 1 and are consistent with $B_{\mathrm{WA}}^{(0)} \approx B_{\mathrm{WA}}^{(1)} \approx B_{\mathrm{WA}}^{(2)}$, but the errors are too large to draw a conclusion concerning WA dilution. Alternatively, the correlation between $\rho_{D}^{3}$ and WA can be studied by considering $\rho_{D}^{3}$ a free parameter in the fit [14]. In this way, the dominant source of uncertainty in the moments is removed. From the variance, first and second moments one obtains very similar constraints linking $\rho_{D}^{3}$ and $B_{\mathrm{WA}}^{(i) s}$. This may be viewed as a mild indication that there is no significant WA dilution, or possibly that the dilution is similar in the three cases. Indeed, assuming vanishing WA at $\mu_{\mathrm{WA}}=0.8 \mathrm{GeV}$ all the moments can be reproduced by $\rho_{D}^{3}(0.5 \mathrm{GeV})=0.05(1) \mathrm{GeV}^{3}$.

In order to connect to $B \rightarrow X_{u} \ell v$ we refer to the WA matrix elements determined in charm decays as $B_{\mathrm{WA}}^{c}$ and consider their relation to those relevant in $B$ semileptonic decays, $B_{\mathrm{WA}}^{b}$, taking into account eq. (3.2). In the heavy quark limit, $f_{P} \sim m_{P}^{-1 / 2}$ [27] so that $B_{P, i}$ scale as constants with heavy quark mass, but recent lattice results give $f_{D} \approx 0.21 \mathrm{GeV}, f_{B} \approx 0.20 \mathrm{GeV}$ [28]. Neglecting any evolution of the WA operators one obtains

$$
B_{\mathrm{wA}}^{b}\left(\mu_{\mathrm{wA}}\right)=\frac{m_{B} f_{B}^{2}}{m_{D} f_{D}^{2}} B_{\mathrm{WA}}^{c}\left(\mu_{\mathrm{WA}}\right) .
$$

The parametric enhancement due to meson masses and decay constants is a significant factor of 2.5. Due to finite heavy quark masses, one might also expect additional power corrections, which spoil the exact scaling of $B_{\mathrm{WA}}$ between the $D$ and $B$ sectors. From eqs. $(4.1,4.2)$ one obtains a bound

$$
\left|B_{\mathrm{WA}}^{b}\left(\mu_{\mathrm{WA}}=0.8 \mathrm{GeV}\right)\right| \lesssim 0.006 \mathrm{GeV}^{3},
$$


which holds for the non-valence contributions, although the lepton moments analysis [14] seems to prefer even smaller values. The valence contribution is more constrained from the ratio of the $D_{s}$ and $D^{0}$ rates and leptonic moments, from which one obtains

$$
-0.004 \mathrm{GeV}^{3} \lesssim \Delta B_{\mathrm{WA}}^{b} \lesssim 0.002 \mathrm{GeV}^{3}
$$

again at $\mu_{\mathrm{WA}}=0.8 \mathrm{GeV}$. The bounds lead to a maximum $2 \%$ WA correction to the total rate of $B \rightarrow X_{u} \ell v$. In turn, this translates into an uncertainty of $1 \%$ on $\left|V_{u b}\right|$ extracted from the total rate and from the most inclusive experimental analyses, like those that involve a lower cut on the invariant hadronic mass. This bound on the WA expectation value improves on previous estimates. In [26], for instance, the maximum value allowed for $B_{\mathrm{WA}}^{b}\left(\mu_{\mathrm{WA}}=1 \mathrm{GeV}\right)$ was as high as $0.020 \mathrm{GeV}^{3}$, or $B_{\mathrm{WA}}^{b}\left(\mu_{\mathrm{WA}}=0.8 \mathrm{GeV}\right)=0.018 \mathrm{GeV}^{3}$.

\section{Summary}

Recent analyses $[11,14]$ have demonstrated that the heavy quark expansion can be employed to describe inclusive semileptonic $D$ decays and to constrain WA effects in the extraction of $\left|V_{u b}\right|$. In addition to the total widths, the Cleo data on the lepton energy spectra have been used to compute the first few moments [14]. The latter are quite sensitive probes of possible WA contributions, both in its isosinglet and isotriplet components, and determine very precisely a linear combination of the expectation values of the Darwin and WA operators. The extraction of WA from the moments depends to the way WA is distributed in the lepton energy spectrum and the effect needs to be taken into account in the error estimates.

The analysis of Cleo data shows no clear evidence for WA, i.e. the OPE describes well the experimental results even in the absence of WA. An upper limit on both valence and non-valence WA components can be derived, which allows to put a bound of $2 \%$ on their relevance in the $B \rightarrow X_{u} \ell v$ decay rate and even less for the isotriplet component.

\section{Acknowledgments}

The author would like to thank Paolo Gambino for a fruitful collaboration as well as the organizers of FPCP 2010 for the invitation to this exciting conference. This work is supported in part by the European Commission RTN network, Contract No. MRTN-CT-2006-035482 (FLAVIAnet) and by the Slovenian Research Agency.

\section{References}

[1] M. Bona et al. [UTfit Collaboration], arXiv:0908.3470 [hep-ph], see also http: //www.utfit.org/; for recent $\left|V_{u b}\right|$ averages see http: //www.slac.stanford.edu/xorg/hfag/.

[2] M. Antonelli et al., arXiv:0907.5386 [hep-ph].

[3] J. Chay, H. Georgi, and B. Grinstein, Phys. Lett. B247 (1990) 399; I. I. Y. Bigi, N. G. Uraltsev, and A. I. Vainshtein, Phys. Lett. B293 (1992) 430 [arXiv:hep-ph/9207214] [Erratum, ibid. B 297 (1993) 477]; I. I. Y. Bigi, M. A. Shifman, N. G. Uraltsev, and A. I. Vainshtein, Phys. Rev. Lett. 71 (1993) 496 
[arXiv:hep- ph/9304225]; A. V. Manohar and M. B. Wise, Phys. Rev. D49 (1994) 1310 [arXiv:hep-ph/9308246].

[4] I. I. Y. Bigi and N. G. Uraltsev, Nucl. Phys. B 423, 33 (1994) [arXiv:hep-ph/9310285].

[5] R. D. Dikeman and N. G. Uraltsev, Nucl. Phys. B 509, 378 (1998) [arXiv:hep-ph/9703437]; I. I. Y. Bigi, R. D. Dikeman and N. Uraltsev, Eur. Phys. J. C 4, 453 (1998) [arXiv:hep-ph/9706520].

[6] I. I. Y. Bigi and N. G. Uraltsev, Z. Phys. C 62, 623 (1994) [arXiv:hep-ph/9311243].

[7] N. Uraltsev, Int. J. Mod. Phys. A 14 (1999) 4641 [arXiv:hep-ph/9905520].

[8] M. B. Voloshin, Phys. Lett. B 515, 74 (2001) [arXiv:hep-ph/0106040].

[9] D. Becirevic, S. Fajfer and J. F. Kamenik, Phys. Lett. B 671, 66 (2009) [arXiv:0804.1750 [hep-ph]].

[10] I. Bigi, T. Mannel, S. Turczyk and N. Uraltsev, arXiv:0911.3322 [hep-ph].

[11] Z. Ligeti, M. Luke and A. V. Manohar, arXiv:1003.1351 [hep-ph].

[12] D. M. Asner et al. [The CLEO Collaboration], arXiv:0912.4232 [hep-ex].

[13] M. Gronau and J. L. Rosner, Phys. Rev. D 79 (2009) 074006 [arXiv:0902.1363 [hep-ph]].

[14] P. Gambino and J. F. Kamenik, Nucl. Phys. B, In Press [arXiv:1004.0114 [hep-ph]].

[15] B. Blok, R. D. Dikeman and M. A. Shifman, Phys. Rev. D 51 (1995) 6167 [arXiv:hep-ph/9410293].

[16] A. Pak and A. Czarnecki, Phys. Rev. Lett. 100, 241807 (2008) [arXiv:0803.0960 [hep-ph]] and K. Melnikov, Phys. Lett. B 666, 336 (2008) [arXiv:0803.0951 [hep-ph]].

[17] A. Czarnecki and M. Jezabek, Nucl. Phys. B 427, 3 (1994) [arXiv:hep-ph/9402326].

[18] V. Aquila, P. Gambino, G. Ridolfi and N. Uraltsev, Nucl. Phys. B 719 (2005) 77 [arXiv:hep-ph/0503083].

[19] M. Gremm and A. Kapustin, Phys. Rev. D 55 (1997) 6924 [arXiv:hep-ph/9603448].

[20] B. M. Dassinger, T. Mannel and S. Turczyk, JHEP 0703, 087 (2007) [arXiv:hep-ph/0611168].

[21] A. K. Leibovich, Z. Ligeti and M. B. Wise, Phys. Lett. B 539, 242 (2002) [arXiv:hep-ph/0205148].

[22] I. I. Y. Bigi, M. A. Shifman, N. Uraltsev and A. I. Vainshtein, Phys. Rev. D 56 (1997) 4017 [arXiv:hep-ph/9704245] and Phys. Rev. D 52 (1995) 196 [arXiv:hep-ph/9405410].

[23] M. Beneke, Phys. Lett. B 434 (1998) 115 [arXiv:hep-ph/9804241].

[24] A. H. Hoang, Z. Ligeti and A. V. Manohar, Phys. Rev. D 59, 074017 (1999) [arXiv:hep-ph/9811239].

[25] A. H. Hoang, Z. Ligeti and A. V. Manohar, Phys. Rev. Lett. 82 (1999) 277 [arXiv:hep-ph/9809423].

[26] P. Gambino, P. Giordano, G. Ossola and N. Uraltsev, JHEP 0710 (2007) 058 [arXiv:0707.2493 [hep-ph]].

[27] B. Grinstein, E. E. Jenkins, A. V. Manohar, M. J. Savage and M. B. Wise, Nucl. Phys. B 380, 369 (1992) [arXiv:hep-ph/9204207].

[28] V. Lubicz and C. Tarantino, Nuovo Cim. 123B, 674 (2008) [arXiv:0807.4605 [hep-lat]]. 